\title{
Promoting Psychological Flexibility on Tolerance Tasks: Framing Behavior Through Deictic/Hierarchical Relations and Specifying Augmental Functions
}

\author{
Bárbara Gil-Luciano ${ }^{1}$ - Francisco J. Ruiz ${ }^{2}$ - Sonsoles Valdivia-Salas ${ }^{3}$. \\ Juan C. Suárez-Falcón ${ }^{4}$
}

(C) Association for Behavior Analysis International 2016

\begin{abstract}
Recent research is advancing in the analysis of the defusion and self-based exercises used in acceptance and commitment therapy (ACT) through relational frame theory (RFT) terms. This study aimed to analyze the effect of two RFT-defined defusion protocols in promoting psychological flexibility by altering the discriminative functions of avoidance of aversive private events. Thirty participants first responded to several questionnaires. Subsequently, participants were exposed to 2 experimental tasks (pretest): a cold pressor and an aversive film. Participants were then randomly assigned to 3 experimental conditions: (a) a control condition, (b) a defusion protocol based on framing one's own behavior through deictic relations (Defusion I), and (c) a defusion protocol that also included hierarchical relations and giving regulatory functions to that discrimination (Defusion II). Finally, participants were again exposed to the 2 experimental tasks (posttest). Results showed that participants who received the defusion protocols performed better in the posttest than did the control participants, and that Defusion II participants showed higher tolerance than Defusion I participants.
\end{abstract}

Bárbara Gil-Luciano

barbaragil.luciano@micpsy.com

1 Madrid Institute of Contextual Psychology, Universidad de Almería, Almería, Spain

2 Fundación Universitaria Konrad Lorenz, Bogotá, Colombia

3 Universidad de Zaragoza, Zaragoza, Spain

4 Universidad Nacional de Educación a Distancia, Madrid, Spain
Keywords Relational frame theory $\cdot$ Acceptance and commitment therapy $\cdot$ Defusion $\cdot$ Self as context $\cdot$ Hierarchical relations

Relational frame theory (RFT; Hayes, Barnes-Holmes, \& Roche, 2001) is a functional-contextual approach to human language and cognition that posits relevant implications for psychological intervention. Most RFT research on this topic has focused on the conceptualization of acceptance and commitment therapy (ACT; Hayes, Strosahl, \& Wilson, 1999) processes (e.g., Barnes-Holmes, Barnes-Holmes, McHugh, \& Hayes, 2004; Luciano et al., 2011; Luciano, ValdiviaSalas, \& Ruiz, 2012; Ruiz \& Luciano, 2015; Törneke, 2010; Törneke, Luciano, Barnes-Holmes, \& Bond, 2016; Villatte, Villatte, \& Hayes, 2016). A recent, relevant advance in this direction has been the RFT conceptualization of psychological flexibility, which is the key concept in ACT.

Psychological flexibility is usually defined in midlevel terms as the ability to stay in the present moment, mindfully aware of private experiences (thoughts, memories, sensations, etc.) and committed to valued goals (Hayes, Luoma, Bond, Masuda, \& Lillis, 2006). However, as described in midlevel terms, the previous definition does not provide clues to improve typical ACT exercises (e.g., defusion and self-based exercises). In this sense, the advantage of an RFT conceptualization of psychological flexibility is that it has the potential to direct attention and research to specific relational behaviors. In RFT terms, psychological flexibility is conceptualized as the generalized repertoire of framing ongoing behavior in hierarchy with the deictic $I$, which typically reduces the discriminative functions of ongoing behavior and allows the derivation of rules that specify appetitive augmental functions and behavior that is in accordance with them (Luciano et al., 2011; Luciano et al., 2012; Luciano, Valdivia-Salas, Cabello, \& 
Hernández, 2009; Ruiz \& Perete, 2015; Törneke et al., 2016). Although the former part of the definition corresponds approximately to the ACT processes of defusion and self-ascontext, the latter part would correspond to the processes of values and committed action.

Consider the following example. Imagine a young taxi driver who is having problems dealing with feelings of anger and irritation. He loves his job, but he cannot help "losing his temper" every time he comes across drivers who make mistakes or drive slowly. He deals with the anger and irritation that arise in these situations by yelling at everyone and driving dangerously, which is affecting him in several ways. According to RFT, this young taxi driver is interacting with his feelings of anger and irritation by responding in coordination with their derived discriminative functions (e.g., yelling at someone, driving dangerously). Psychological flexibility here would imply a movement in which he would learn to notice his anger, observe it, realize he is the one who is watching it (i.e., defusion and self-based exercises in midlevel terms), and act according to what is really important to him in the long term (i.e., values). In RFT terms, framing his thoughts and anger in hierarchy with the deictic $I$ so that other sources of stimulus control, such as the appetitive augmentals related to his desire to be a calm and safe driver, can enter the stage, and actions coordinated with these rules can emerge.

Some predictions can be suggested following the RFT definition of psychological flexibility to improve defusion and self-as-context exercises in ACT. According to RFT, the most basic processes involved in these exercises are the discrimination of one's own ongoing behavior through a hierarchical relation with the deictic $I$ and the specification of appetitive augmental rules (i.e., providing regulatory functions to the discrimination). Thus, exercises that promote all these processes should be more efficacious than those that do not.

So far, empirical investigations have found that framing ongoing behavior only through a deictic relation (I-HERE, Behavior-THERE) is less effective than including explicit hierarchical relations (I CONTAIN Behavior) and the specification of appetitive augmental rules (i.e., regulatory functions). In the pioneering study, Luciano et al. (2011) studied the effect of two protocols with adolescents with high scores on impulsivity or emotional symptoms. The Defusion I protocol contained trials consisting of framing ongoing behavior through a deictic relation, whereas the Defusion II protocol was the same but added explicit hierarchical cues and questions directed to specify appetitive augmentals. The findings showed that the Defusion II protocol had a greater effect on reducing the frequency of problematic behaviors and psychological inflexibility at the 4-month follow-up.
Following the previous study, Foody, Barnes-Holmes, Barnes-Holmes, and Luciano (2013) presented an analog study in which participants were first exposed to a distressinduction task consisting of writing and saying aloud a negative self-referential thought. Afterwards, participants were randomly allocated to two experimental conditions. In one condition, participants received a protocol consisting of framing behavior through a deictic relation that the authors called "distinction self as context" protocol. In the other condition, the protocol included hierarchical relations and interactions to provide regulatory functions to the discrimination (called "hierarchical self as context" protocol). The latter protocol was more efficacious in reducing experimentally induced emotional distress. In a similar subsequent study, Foody, BarnesHolmes, Barnes-Holmes, Rai, and Luciano (2015) also found that protocols that included framing ongoing thoughts through a hierarchical relation were more efficacious than those that only presented deictic relations.

In summary, the three studies to date showed that protocols that incorporate explicit hierarchical relations and regulatory functions to the discrimination of ongoing behavior are more efficacious than protocols that only include deictic relations. Nonetheless, none of these studies used behavioral tasks as a way to obtain direct measures of psychological flexibility. Whereas in Luciano et al. (2011), the dependent variables were self-reports of the frequency of problematic behaviors and psychological inflexibility, in Foody et al. (2013), they were self-reports of experienced discomfort. This is a limitation because the aim of defusion and self-based exercises is to change the discriminative functions of ongoing behavior so that the individual can behave in a valued way, even in the presence of discomfort. In this sense, the differential effect of the relational framings involved in these exercises is more precisely analyzed with behavioral measures.

To advance in the abovementioned direction, the current study aimed to replicate and extend the previous findings by comparing the effect of three conditions on increasing tolerance to the aversive functions induced by a cold pressor and an aversive film task. Two conditions included defusion-based protocols similar to the ones used in Luciano et al. (2011) and Foody et al. (2013). Specifically, Defusion I consisted of multiple-exemplar training in framing ongoing behavior through a deictic relation (I-HERE, Behavior-THERE), and Defusion II incorporated hierarchical framing (I CONTAIN Behavior) and regulatory functions to this discrimination. A control condition was also included to explore the efficacy of the two protocols compared to no intervention. As compared to previous studies (Luciano et al., 2011; Foody et al., 2013; Foody et al., 2015), this study supposes two relevant advances. First, we implemented a control condition that permits the isolation of the effect of Defusion I protocol. Second, the main dependent variable was a behavioral measure (i.e., discomfort tolerance). 


\section{Method}

\section{Participants}

Thirty-one adults volunteered to participate in the study. One participant was excluded due to having reached the maximum pain tolerance during the pretest phase of the cold pressor task (see Experimental Tasks section). The final sample consisted of 30 participants ( 15 men, 15 women) between the ages of 21 and 46 years $(M=26.8$ years, $S D=5.5)$.

\section{Setting and Apparatus}

All sessions were conducted individually in an experimental room equipped with a table, two chairs, an armchair, a personal computer, headphones, a $40 \times 30 \times 25 \mathrm{~cm}$ plastic container filled with water and ice, and a digital thermometer adhered to the container that was only visible for the experimenter.

\section{Experimental Tasks}

Cold pressor In this task, participants were invited to submerge their right arm up to their elbow in a plastic container with ice water at 4 degrees Celsius. Even though they were requested to leave their arm in the water for as long as possible, they were also reminded that they were free to stop and remove their arm from the water at any time. Tolerance to pain was then measured by the total amount of time participants remained with their arm in the water. Participants who left their arm in the water for 300 seconds in their first exposure to the task were excluded from further participation in the study because they reached the maximum pain tolerance admissible for ethical reasons.

Aversive film This task consisted of the presentation of an aversive, 90-second film identical to those used in López et al. (2010). Two films were used that contained images of surgical procedures (amputation of limbs) and were presented without audio; however, participants were invited to use the headphones so that they could focus better on the viewing. As in the first task, the experimenter asked participants to continue to watch the film on the computer for as long as possible, but also reminded them that they were free to interrupt the task wherever they wanted. Tolerance to the video was measured by the total amount of time participants continued to watch the film. The task was considered completed when participants chose to stop the film or when the experimenter noticed that they looked away from the center of the screen.

\section{Design and Variables}

Before beginning the experiment, participants were randomly assigned to one of three experimental conditions (control, Defusion I, and Defusion II), with the only restriction of balancing sex (5 men and 5 women per condition). Each condition consisted of 10 participants. The main dependent variable was tolerance both in the cold pressor and aversive film tasks. An additional dependent variable was the discomfort reported by the participants in both tasks. The independent variable was the protocol to which participants were exposed after the pre-intervention presentation of the experimental task.

Participants in the control condition only underwent a general interview with the experimenter unrelated to the experimental procedure. Participants in Defusion I received a defusion protocol that included multiple-exemplar training in framing ongoing private experiences through deictic relations. Similar to previous studies (e.g., Luciano et al., 2011; Ruiz, Luciano, Vizcaíno-Torres, \& Sánchez, 2012; Ruiz \& Perete, 2015), the protocol advanced from trials with neutral experiences to trials with aversive experiences related to both experimental tasks. Participants in Defusion II received the same protocol as in Defusion I, but including interactions that promoted the framing of ongoing experiences through explicit hierarchical relations and the promotion of regulatory functions to that discrimination. All protocols lasted approximately 30 minutes.

\section{Instruments and Measures}

Acceptance and Action Questionnaire--II (AAQ-II; Bond et al., 2011; Spanish version by Ruiz, Langer, Luciano, Cangas, \& Beltrán, 2013). The AAQ-II is a general measure of experiential avoidance. It consists of 7 items that are rated on a 7-point Likert-type scale $(7=$ always true; $1=$ never true $)$. The Spanish version of the AAQ-II has shown good psychometric properties (mean alpha of .88) and a one-factor structure.

Cognitive Fusion Questionnaire (CFQ; Gillanders et al., 2014; Spanish version by Ruiz, Suárez-Falcón, RiañoHernández, \& Gillanders, 2017, in press). The CFQ is a 7item, 7-point Likert-type scale ( 7 = always true; 1 = never true $)$ consisting of sentences describing instances of cognitive fusion. The Spanish version of the CFQ has shown good psychometric properties (mean alpha of .91) and a one-factor structure.

Depression, Anxiety, and Stress Scales-21 (DASS-21; Antony, Bieling, Cox, Enns, \& Swinson, 1998; Spanish version by Daza, Novy, Stanley, \& Averill, 2002). The DASS-21 is a 21-item, 4-point Likert-type scale ( $3=$ applies to me very 
much, or most of the time; $0=$ does not apply to me at all) consisting of sentences describing negative emotional states. It contains three subscales: Depression, Anxiety, and Stress. The Spanish version has shown good psychometric properties (alpha of the complete scale of .90) and a three-factor structure.

Self-reports of pain and discomfort during experimental tasks Self-reports of experienced pain (cold pressor) or discomfort (aversive film) after each experimental task were obtained by asking the participants to respond to visual analogue scales (VAS) on which they had to indicate a score from 0 (no pain or discomfort) to 100 (unbearable pain or discomfort).

\section{Protocols}

Control protocol Participants in the control condition had no intervention. Instead, a general interview took place between the experimenter and the participants, with general questions about what they studied or what their job was, whether they liked it, what were their future plans, and so on. The conversation was not related to the experimental tasks or the study.

Defusion protocols Both conditions (Defusion I and II) were administered in two parts. Protocols were presented as follows (the italicized sentences belong to what was added to the Defusion II protocol):

\section{Part I: Multiple-exemplar training using neutral private} events. The experimenter said: "I would like you to close your eyes and listen to what I say. If you suddenly find yourself distracted from the exercise, just say so and we will go back to where you were before the distraction. For now, just focus on your breath. See if you can notice your belly rising every time you inhale . . . and how it falls every time you exhale. Ask yourself, who is breathing? Who is noticing his/her belly rising ... and then falling? Now, see if you can picture your belly as if you had a bag that inflates every time you inhale ... and that deflates when you exhale. Ask yourself, who is picturing his/her belly like a bag that inflates ... and deflates? Can you realize you are the one watching it like that? Now, try to focus on the posture you are maintaining. Go over your arms ... your legs . . . tell me, which part of your body has the most comfortable posture? ( . . . ) Notice that comfort. ... See if you can give it a shape ... a color ... don't do anything with it, just contemplate it. Ask yourself, who is noticing that comfort there? Can you realize you are the one contemplating it? Now, go over your general posture again and tell me which part of your body feels a bit uncomfortable. ( . . .) Ok, just notice the discomfort. ... See if you can give it a shape . . . a color ... don't do anything with it, just contemplate it. Ask yourself, who is noticing that discomfort there? Can you realize you are the one contemplating it? Imagine yourself doing whatever you would do if you let that discomfort be in charge of what you do: Imagine yourself changing your posture so that the discomfort is gone. Now, imagine that you are the one in charge of what you do and not that discomfort: imagine yourself remaining in the posture, making room for the discomfort."

This procedure was conducted with some bodily sensations that were present for the participant in that moment. Then, the experimenter continued: "Now, go over your mind and the thoughts it is giving you right now. See how thoughts just show up. Let's pick any of them. Can you say one of them out loud? ( . . . Great, imagine you write it on a balloon. Can you see it written there? Don't do anything with it, just let it be there. Tell me, who is contemplating that thought there, written on the balloon? Do you realize that you can watch the thought there? Now, let's grab any other thought your mind is giving you right now...."

The same procedure was repeated with three more thoughts. If they were aversive to the participant and he or she was in the Defusion II condition, they were asked to imagine themselves doing whatever they would do if they let the thought be in charge of what they do, followed by asking them to imagine what they would do if they were in charge of what they do: "Imagine yourself pushing away the thought, or arguing with it. . . Now, see if you can imagine yourself just letting that thought stay there, not doing anything with it." After three thoughts were dealt with, independently of their nature, participants in Defusion II condition were instructed to do the following: "Allow yourself to be much bigger than all these thoughts that are here with you ... realize that you have enough room to have whatever thoughts that might show up."

\section{Part II: Multiple-exemplar training using aversive private} events In Part II, we specifically asked participants to go back to the moment they had undergone the experimental tasks. The experimenter said: "Now, I would like you to go back to the moment before you placed your hand and forearm in the water container. ... Try to go back to that moment. See if you recall my voice saying 'Now' and then see yourself placing your hand and forearm in the water. What did you feel just at that moment? What sensation did you have in your hand? ( . . I Imagine that you can feel that kind of pin-pricks all over your hand and forearm. . . . Can you? Now, ask yourself who is imagining that sensation over there? Imagine you can take a picture of that pain in your hand ... take it and put it in front of you. Who is contemplating that picture of pain in the hand? Now, imagine yourself letting that pain be in charge of what you do. See yourself doing whatever you would do if the pain were in charge. What would you do, then? (. . ) Now, imagine that you allow yourself to be in charge of the situation. Ask yourself what would you do if you were in charge (...) Would you be bigger than your pain in that case? Imagine that you give yourself the chance to place yourself over your pain. Imagine 
you apply this to any sort of situation in your daily life, when you feel something you don't want to feel."

The same procedure was repeated with several physical sensations and thoughts that the participant reported having felt during the cold pressor task. Then, the whole process was repeated with the physical sensations and thoughts experienced during the aversive film task.

\section{Procedure}

The procedure of the study was approved by the Center for Psychological Research of the Fundación Universitaria Konrad Lorenz. The experimental sessions were conducted individually and lasted approximately 75 minutes, distributed in four phases (see Fig. 1).

Phase 1. Preexperimental Mmeasures The first 15 minutes were used to obtain the pretest measures, which were preceded by an informed consent to exclude participants who reported some medical history incompatible with the cold pressor task, such as heart or circulation problems (e.g., Raynaud's syndrome), blood pressure problems, diabetes, epilepsy, and recent serious injury. To make the experimental tasks valuable to them, participants were told that the aim of the study was to analyze what kind of coping strategies might be helpful to

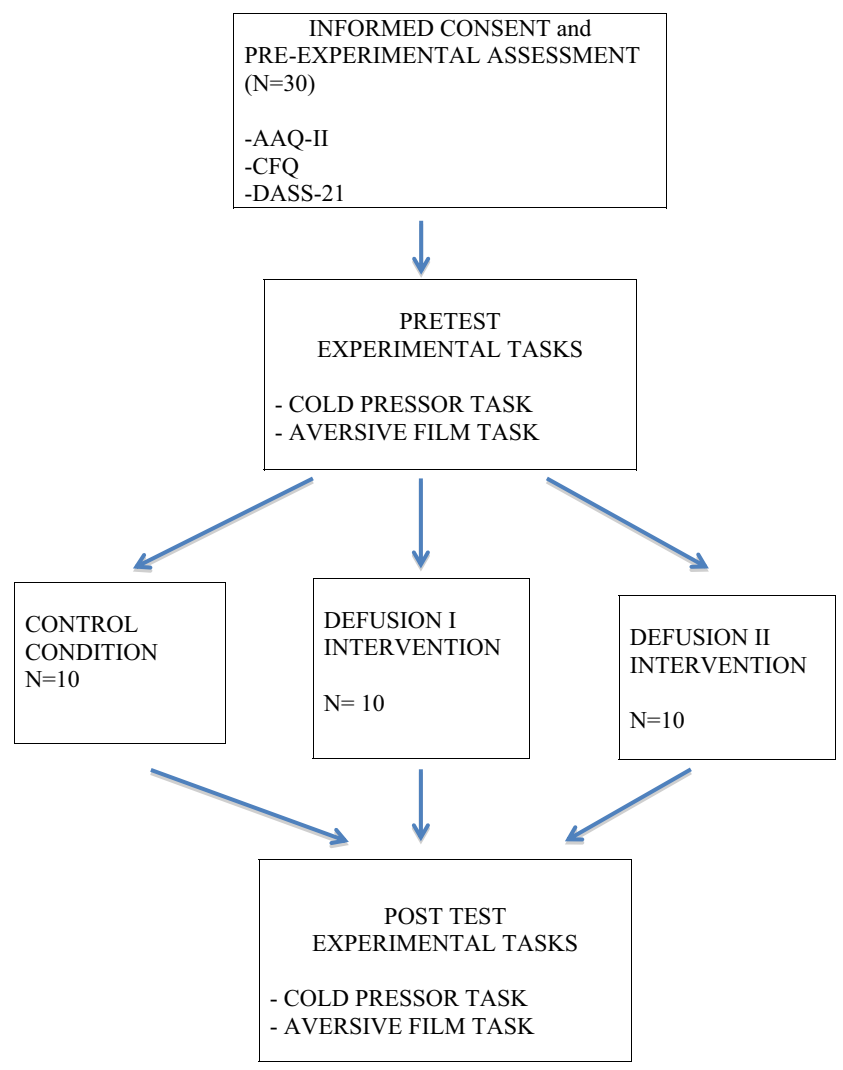

Fig. 1 Overview of the experimental procedure people suffering from constant pain and/or who have to deal with situations that are accompanied by much discomfort. Then, participants responded to the AAQ-II, CFQ, and DASS-21.

Phase 2. Experimental Tasks I (Pretest) Participants were first exposed to the cold pressor task. After a brief pause, the aversive film task commenced.

Phase 3. Protocols Participants allocated either to the Defusion I or II condition were asked to sit in the armchair and to remain seated there, eyes closed, during the following exercises. They were told that these exercises had the aim of teaching them some strategies to cope with pain or discomfort during the second round of tasks, so that they could try to perform them for a longer period of time. Defusion I and II interventions lasted approximately 20-30 minutes. There was only one experimenter (a trained ACT therapist) conducting the interview for the control condition and administering the defusion interventions.

Phase 4. Experimental Tasks II (Posttest) A second exposure to the tasks took place after the implementation of the protocols. This phase was identical to the first exposure to the tasks.

\section{Data Analysis}

Statistical analyses were performed on SPSS 20. Analyses of variance (ANOVAs) were first conducted to explore the equivalence of the experimental conditions on age and pretest tolerance and intensity of pain/discomfort in both the cold pressor and aversive film tasks (Phase 2). Subsequently, additional ANOVAs were computed on the change scores (posttest scorepretest score ) to analyze the differential effect of the protocols. The proportions of the total variance that is attributed to an effect (i.e., eta squared, or $\eta^{2}$ ) are reported as effect sizes typical of ANOVA. Eta squared values of .01, .06, and .14 were interpreted as small, medium, and large effects, respectively. Last, when the assumption of equal variances to conduct ANOVAs was not met, we computed the Welch's robust test.

After conducting ANOVAs, planned contrasts were conducted to test whether (a) the change scores on tolerance of participants in the Defusion II condition were higher than those in the Defusion I and control conditions and (b) the change scores on tolerance in Defusion I were higher than those in the control condition. These contrasts were one-tailed tests and interpreted with a significance level of $p<.05$. Between-condition effect sizes in Phase 4 were calculated with Cohen's $d$ (Cohen, 1988), which can be interpreted as small ( $d=.20$ to .49$)$, medium $(d=.50$ to .79$)$, and large (above $d=.80)$.

Moderation analyses were conducted with the nonparametric bootstrapping procedure to estimate conditional effects 
using the PROCESS package (Hayes, 2013). Interaction effects were deemed significant if the $95 \%$ bias-corrected (BC) bootstrap confidence intervals $(\mathrm{CI})$ for those effects based on 20,000 bootstrapped samples did not include zero. Simple moderation analyses were conducted to analyze whether cognitive fusion scores (i.e., CFQ), age, and sex acted as moderator variables of the effect of the experimental protocols.

\section{Results}

\section{Initial Equivalence of Experimental Conditions}

ANOVAs revealed no significant differences at pretest among the groups for age, tolerance, and intensity of pain/discomfort measures in the two experimental tasks (see Table 1). There were no differences among conditions in the AAQ-II (experiential avoidance), CFQ (cognitive fusion), and DASS-21 (emotional symptoms), and $63 \%$ of the participants viewed the complete film in Phase $2(6,6$, and 7 for the control condition, Defusion I, and Defusion II, respectively).

\section{Effects of the Experimental Protocols}

Table 2 shows the descriptive statistics of the pre-post change scores in tolerance and intensity/discomfort in the two experimental tasks and the results from ANOVAs.

Tolerance Figure 2 shows the pre-post change in tolerance both in the cold pressor and aversive film tasks. The conducted ANOVA revealed that there were statistically significant differences between conditions in relation to the pre-post change in the cold pressor task, $F(2,16)=4.94, p<.05, \eta^{2}=.23$. Planned contrasts revealed that participants in the Defusion II condition significantly increased tolerance compared to participants both in the Defusion I $(t=2.032, p<.05, d=.91)$ and control conditions $(t=2.593, p=.02, d=1.16)$. Likewise, participants in

Table 1 Descriptive Data for Each Condition at Pretest and ANOVA Results

\begin{tabular}{llllll}
\hline & Control & Defusion I & Defusion II & $F(d f)$ & $p$ \\
\hline Age & 27.90 & 27.50 & 25.00 & .81 & .46 \\
& $(7.26)$ & $(5.04)$ & $(3.71)$ & $(2,27)$ & \\
Tolerance Cold pressor & 42.60 & 38.50 & 60.30 & .98 & .39 \\
& $(30.62)$ & $(21.39)$ & $(52.12)$ & $(2,27)$ & \\
Intensity Cold pressor & 75.00 & 70.50 & 71.50 & .39 & .68 \\
& $(9.43)$ & $(16.91)$ & $(7.09)$ & $(2,27)$ & \\
Tolerance Aversive film & 81.69 & 73.13 & 62.89 & .57 & .57 \\
& $(29.76)$ & $(38.05)$ & $(47.99)$ & $(2,27)$ & \\
Intensity Aversive film & 50.50 & 56.50 & 66.50 & .66 & .53 \\
& $(26.71)$ & $(36.71)$ & $(29.82)$ & $(2,27)$ & \\
\hline
\end{tabular}

Standard deviations in parentheses
Defusion I significantly increased tolerance compared to the Control condition $(t=2.25, p<.05, d=1.01)$.

In relation to the aversive film task, the differences between conditions were marginally significant although the effect size was almost large, $F(2,27)=3.21, p=.06, \eta^{2}=.132$. Planned contrasts revealed that participants in Defusion II significantly increased tolerance compared to the control participants $(t=2.50, p<.05, d=1.19)$. The differences between Defusion II and Defusion I were marginally significant favoring the former condition $(t=1.59, p=.06, d=.65)$. There were no significant differences between Defusion I and control conditions $(t=.91, p>.05, d=.43)$.

Pain/Discomfort There were no statistically significant differences between conditions in the pre-post change either in perceived pain during the cold pressor task, $F(2,27)=.10$, $p>.05, \eta^{2}=.01$, or in the discomfort experience during the aversive film, $F(2,27)=2.09, p>.05, \eta^{2}=.07$.

\section{Moderation Analyses}

Cognitive fusion as measured by the CFQ was a statistically significant moderator of the effect of the experimental protocols on the change score in the aversive film (incremental $R^{2}=.20, p=.007$ ), whereas it was a marginally significant moderator in relation to the cold pressor (incremental $R^{2}=.07, p=.09$ ). Figure 3 shows the pre-post change scores on both experimental tasks at percentiles on the CFQ for each experimental condition according to the regression model. It can be observed that the differences across conditions are greater as a function of participants' cognitive fusion levels.

As expected, age and sex were not significant moderators of the effect of the experimental protocols (age with cold pressor: incremental $R^{2}=.003, p=.46$; age with aversive film: incremental $R^{2}=.016, p=.75$; sex with cold pressor: incremental $R^{2}=.002, p=.18$; sex with aversive film: incremental $R^{2}=.048, p=.79$ ).

\section{Discussion}

This study aimed to analyze the differential effect of two ACTbased protocols derived from the RFT definition of psychological flexibility (Törneke et al., 2016). Specifically, the first protocol consisted of multiple-exemplar training in framing ongoing private experiences through deictic relations, whereas the second protocol added framing ongoing private events through hierarchical relations and questions directed to specify appetitive augmental functions (i.e., provide regulatory functions to the discrimination of ongoing experiences). Because previous research has named these interventions, respectively, Defusion I and Defusion II (Luciano et al., 2011; 
Table 2 Descriptive Data for Pre-Post Change in Each Condition and ANOVA Results

\begin{tabular}{lllllll}
\hline & Control & Defusion I & Defusion II & $F(d f)$ & $p$ & $\eta^{2}$ \\
\hline Tolerance Cold pressor & -10.30 & .60 & 39.10 & 4.94 & .021 & .23 \\
& $(11.72)$ & $(9.83)$ & $(59.10)$ & $(2,27)$ & & \\
Tolerance Aversive film & -14.82 & -4.42 & 13.73 & 3.21 & .06 & .13 \\
& $(19.53)$ & $(28.13)$ & $(27.94)$ & $(2,27)$ & & \\
Intensity Cold pressor & 5.00 & 6.50 & 4.50 & .10 & .91 & .01 \\
& $(7.07)$ & $(13.13)$ & $(10.65)$ & $(2,27)$ & & \\
Intensity Aversive film & 12.00 & 6.00 & -4.50 & 2.09 & .14 & .07 \\
& $(19.60)$ & $(21.57)$ & $(12.34)$ & $(2,27)$ & & \\
\hline
\end{tabular}

Standard deviations in parentheses
Foody et al., 2013), we followed this nomenclature. However, it is worth noting that alternative names such as Flexibility I and Flexibility II could be more appropriate.

The effects of the two protocols and a control condition were tested in two behavioral tasks that involved discomfort tolerance. The results showed that participants in Defusion II significantly increased their pain tolerance in the cold pressor task in comparison to participants in Defusion I and control condition. Likewise, participants in Defusion I showed higher pain tolerance than control participants. The results in the aversive film task followed a similar pattern. Defusion II participants showed statistically significant greater tolerance than did control participants and marginally significant greater tolerance than did participants in Defusion I. There was no statistically significant difference between participants in Defusion I and control participants.
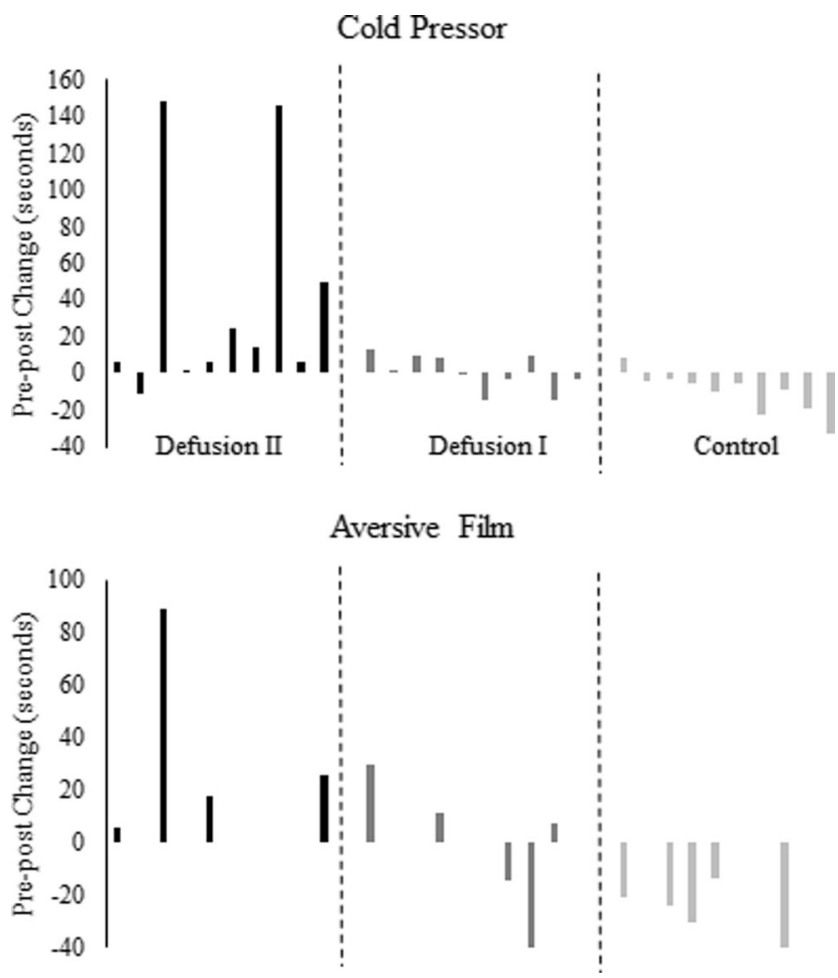

Fig. 2 Pre-post change in the experimental tasks in each condition. Blank spaces represent no pre-post change
No significant change took place in the intensity of pain and discomfort from pretest to posttest in any of the two tasks. This suggests that the process of change of the defusion protocols, especially in Defusion II, was the alteration of the discriminative functions for avoiding discomfort rather than the discomfort decrease. In other words, the defusion protocols seemed to promote more flexible reaction (i.e., psychological flexibility) to the discomfort induced by the experimental tasks. These results are consistent with the study conducted by Gutiérrez, Luciano, Rodríguez, and Fink (2004), where it was found that participants in the ACT condition continued to perform the pain task even though they reported high levels of pain. In line with this, it is worth mentioning that the higher the participant's level

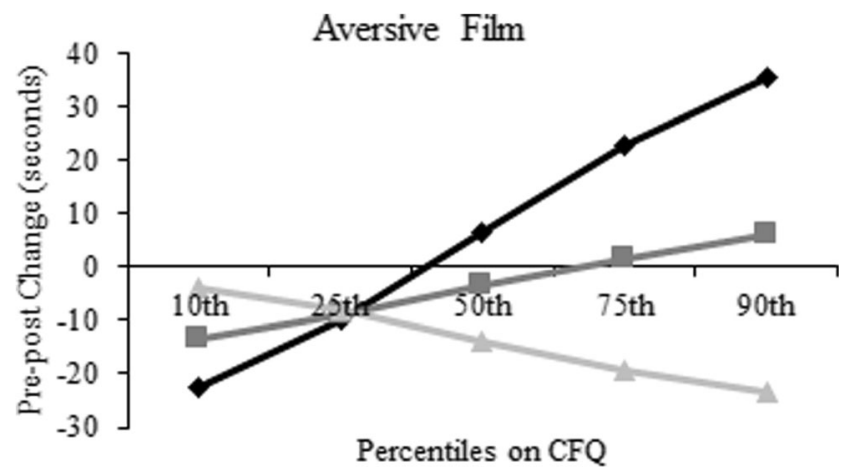

Cold pressor

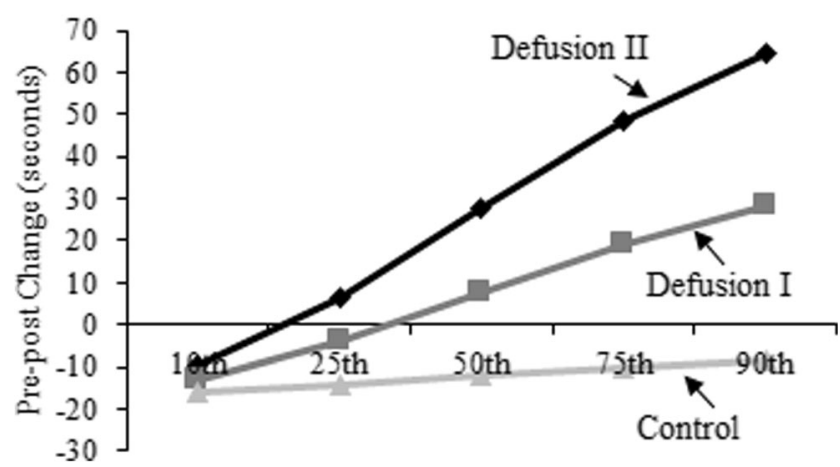

Fig. 3 Pre-post change scores on the cold pressor and aversive film tasks for each experimental condition as a function of percentiles on the CFQ, according to the moderation analysis 
of cognitive fusion, the more Defusion II and I protocols seemed to affect the performance of the tasks (especially in the aversive film task) as compared to the control condition. In other words, the defusion protocols were more effective in participants who showed a pattern of behavior in coordination to the discriminative functions of aversive private events (i.e., psychological inflexibility) as measured by the CFQ. This is consistent with the aim of the defusion protocols to the extent that participants showing more fused behavior with discomfort would be the ones with more potential to improve their tolerance to pain and discomfort.

Some limitations of the study are worth mentioning. First, even though we separated deictic from hierarchical cues, it would be relevant to separate the different cues involved in the Defusion II protocol, that is, the hierarchical cues from the questions aimed at providing regulatory functions to the discrimination of one's behavior. Accordingly, the interactions responsible for the change in the participants' behavior still remain unclear. Second, the aversive film task was not a standardized task, and it was found that many participants reached the maximum level of tolerance in the pretest as well as in the posttest. In other words, for many participants, it was not a task that produced a relevant dose of discomfort. In those cases, the task was not a good alternative to measure changes in the discriminative functions of discomfort.

This is the first study that has analyzed the effect of different types of relational framing in tolerance to discomfort induced by experimental tasks. The results showed that framing ongoing discomfort through deictic and hierarchical relations plus providing regulatory functions (i.e., specifying appetitive augmental functions) to this discrimination produce greater decrease of the discomfort's discriminative functions than framing the experience only through deictic relations. This is consistent with the study by Luciano et al. (2011), in which the Defusion II protocol showed a greater effect in reducing the frequency of participants' problematic behaviors and psychological inflexibility. The results are also consistent with the studies by Foody et al. (2013; Foody et al., 2015) that found the superiority of adding hierarchical cues and regulatory functions to decrease the discomfort experienced after a distress induction task. On the one hand, as compared to Luciano et al. (2011), this study added greater experimental control by (a) randomly assigning the participants to the experimental conditions, (b) implementing a control condition that did not receive an active protocol that permitted analyzing the effect of Defusion I for the first time, and (c) relying on behavioral measures rather than on participants' responses to self-reports. On the other hand, as compared to the studies by Foody et al. (2013; Foody et al., 2015), this study can be seen as a better demonstration of the effect of different types of defusion or self-based exercises because their primary aim is to change the discriminative function of discomfort and not necessarily the discomfort itself.
In conclusion, this study adds further empirical evidence of the effect of emphasizing hierarchical relations and cues for effective regulation in increasing the effect of defusion exercises that consist of solely framing ongoing behavior through deictic relations. The results of this study are more directly translatable to problems in which increasing tolerance to discomfort could be a therapeutic aim, such as in chronic pain or in individuals under physiotherapy.

\section{Compliance with Ethical Standards}

Ethical approval All procedures performed in this study were in accordance with the ethical standards of the institutional and/or national research committee and with the 1964 Helsinki Declaration and its later amendments or comparable ethical standards.

Conflict of Interest The authors declare that they have no conflict of interest.

Informed consent Informed consent was obtained from all individual participants included in the study.

Funding This study was supported, in part, by funds from the research projects PSI2011-25497 (Ministerio de Ciencia e Innovación) and PSI2014-59610-P (Ministerio de Economía y Competitividad).

\section{References}

Antony, M. M., Bieling, P. J., Cox, B. J., Enns, M. W., \& Swinson, R. P. (1998). Psychometric properties of the 42 -item and 21 -item versions of the Depression Anxiety Stress Scales (DASS) in clinical groups and a community sample. Psychological Assessment, 10, 176-181. doi:10.1037/1040-3590.10.2.176.

Barnes-Holmes, Y., Barnes-Holmes, D., McHugh, L., \& Hayes, S. C. (2004). Relational frame theory: Some implications for understanding and treating human psychopathology. International Journal of Psychology and Psychological Therapy, 4, 355-375.

Bond, F. W., Hayes, S. C., Baer, R. A., Carpenter, K. M., Guenole, N., Orcutt, H. K., ... Zettle, R. D. (2011). Preliminary psychometric properties of the Acceptance and Action Questionnaire-II: A revised measure of psychological inflexibility and experiential avoidance. Behavior Therapy, 42, 676-688. doi:10.1016/j.beth.2011.03.007

Cohen, J. (1988). Statistical power analysis for the behavioral sciences (2nd ed.). Hillsdale: Erlbaum.

Daza, P., Novy, D. M., Stanley, M., \& Averill, P. (2002). The Depression Anxiety Stress Scale-21: Spanish translation and validation with a Hispanic sample. Journal of Psychopathology and Behavioral Assessment, 24, 195-205. doi:10.1023/A:1016014818163.

Foody, M., Barnes-Holmes, Y., Barnes-Holmes, D., Rai, L., \& Luciano, C. (2015). An empirical investigation of the role of self, hierarchy, and distinction in a common act exercise. The Psychological Record, 65, 231-243. doi:10.1007/s40732-014-0103-2.

Foody, M., Barnes-Holmes, Y., Barnes-Holmes, D., \& Luciano, C. (2013). An empirical investigation of hierarchical versus distinction relations in a self-based ACT exercise. International Journal of Psychology and Psychological Therapy, 13, 373-385.

Gillanders, D. T., Bolderston, H., Bond, F. W., Dempster, M., Flaxman, P. E., Campbell, L., ... Remington, B. (2014). The development and 
initial validation of the Cognitive Fusion Questionnaire. Behavior Therapy, 45, 83-101. doi:10.1016/j.beth.2013.09.001

Gutiérrez, O., Luciano, C., Rodríguez, M., \& Fink, B. C. (2004). Comparison between an acceptance-based and a cognitive-controlbased protocol for coping with pain. Behavior Therapy, 35, 767 783. doi:10.1016/S0005-7894(04)80019-4.

Hayes, A. F. (2013). Introduction to mediation, moderation, and conditional process analysis. A regression-based approach. New York: Guilford Press.

Hayes, S. C., Barnes-Holmes, D., \& Roche, D. (Eds.). (2001). Relational frame theory: A post-Skinnerian account of human language and cognition. New York: Plenum Press.

Hayes, S. C., Luoma, J. B., Bond, F. W., Masuda, A., \& Lillis, J. (2006). Acceptance and commitment therapy: Model, processes and outcomes. Behaviour Research and Therapy, 44, 1-25. doi:10.1016/j. brat.2005.06.006.

Hayes, S. C., Strosahl, K. D., \& Wilson, K. G. (1999). Acceptance and commitment therapy. An experiential approach to behavior change. New York: Guilford.

López, J. C., Ruiz, F. J., Feder, J., Barbero-Rubio, A., Suárez-Aguirre, J., Rodríguez, J. A., \& Luciano, C. (2010). The role of experiential avoidance in the performance on a high cognitive demand task. International Journal of Psychology and Psychological Therapy, $10,475-488$.

Luciano, C., Ruiz, F. J., Vizcaíno-Torres, R. M., Sánchez-Martín, V., Gutiérrez-Martínez, O., \& López-López, J. C. (2011). A relational frame analysis of defusion interactions in acceptance and commitment therapy: A preliminary and quasi-experimental study with atrisk adolescents. International Journal of Psychology and Psychological Therapy, 11, 165-182.

Luciano, C., Valdivia-Salas, S., Cabello, F., \& Hernández, M. (2009). Developing self-directed rules. In R. A. Rehfeldt \& Y. BarnesHolmes (Eds.), Derived relational responding: Applications for learners with autism and other developmental disabilities (pp. 335-352). Oakland: New Harbinger.
Luciano, C., Valdivia-Salas, S., \& Ruiz, F. J. (2012). The self as the context for rule-governed behavior. In L. McHugh \& I. Stewart (Eds.), The self and perspective taking: Research and applications (pp. 143-160). Oakland: Context Press.

Ruiz, F. J., Langer, A. I., Luciano, C., Cangas, A. J., \& Beltrán, I. (2013). Measuring experiential avoidance and psychological inflexibility: The Spanish translation of the Acceptance and Action Questionnaire. Psicothema, 25, 123-129. doi:10.7334 /psicothema2011.239.

Ruiz, F. J., \& Luciano, C. (2015). Common physical properties among relational networks improve analogy aptness. Journal of the Experimental Analysis of Behavior, 103, 498-510. doi:10.1002/jeab.147.

Ruiz, F. J., Luciano, C., Vizcaíno-Torres, R. M., \& Sánchez, V. (2012). Aplicación de la terapia de aceptación y compromiso (ACT) en trastornos de ansiedad en la infancia. Un caso de fobia a la oscuridad [Application of acceptance and commitment therapy (ACT) in children with anxiety disorders: A case study of darkness phobia]. In M. Páez \& O. Gutiérrez (Eds.), Múltiples aplicaciones de la terapia de aceptación y compromiso [Multiple applications of acceptance and commitment therapy] (pp. 27-44). Madrid: Pirámide.

Ruiz, F. J., \& Perete, L. (2015). Application of a relational frame theory account of psychological flexibility in young children. Psicothema, 27, 114-119. doi:10.7334/psicothema2014.195.

Ruiz, F. J., Suárez-Falcón, J. C., Riaño-Hernández, D., \& Gillanders, D. (2017, in press). Psychometric properties of the Cognitive Fusion Questionnaire in Colombia. Revista Latinoamericana de Psicología.

Törneke, N. (2010). Learning RFT: An introduction to relational frame theory and its clinical applications. Oakland: Context Press.

Törneke, N., Luciano, C., Barnes-Holmes, Y., \& Bond, F. (2016). RFT for clinical practice: Three core strategies in understanding and treating human suffering. In R. D. Zettle, S. C. Hayes, D. BarnesHolmes, \& A. Biglan (Eds.), The Wiley handbook of contextual behavioral science (pp. 254-273). New York: Wiley Blackwell.

Villatte, M., Villatte, J. L., \& Hayes, S. C. (2016). Mastering the clinical conversation: Language as intervention. New York: Guilford Press. 